\title{
PROFIL-2 Experiment and neutron capture cross sections of Europium iso- topes
}

\author{
Shengli Chen ${ }^{1,2, *}$, Gilles Noguere ${ }^{1}$, David Bernard ${ }^{1}$, Cyrille De Saint Jean ${ }^{1}$, and Jean Tommasi ${ }^{1}$ \\ ${ }^{1}$ CEA, Cadarache, DEN/DER/SPRC/LEPh, 13108 Saint Paul Les Durance, France \\ ${ }^{2}$ I-MEP2, University Grenoble Alpes, 38402 Saint Martin d'Heres, France
}

\begin{abstract}
Neutron-induced cross section is one of the key quantities in nuclear physics and nuclear engineering. The integral experiment can give good feedback to the cross sections with low uncertainties. Using the optical model and statistical model, the neutron-induced total and capture cross sections of ${ }^{153}$ Eu are revaluated according to the experimental microscopic total cross sections and the PROFIL-2 integral experiment. The corresponding uncertainties and covariances are determined with the data assimilation method implemented in CONRAD code. On the other hand, the previous interpretation of the PROFIL-2 experiment showed that JEFF-3.1 overestimates the neutron-induced capture cross section of ${ }^{151} \mathrm{Eu}$ by a factor of 2 . Further analysis performed in the present work points out that the large difference between calculation and experimental data is mainly due to the lack of ${ }^{152 \mathrm{~m} 1} \mathrm{Eu}$ in ERANOS code, which was used to interpret the PROFIL-2 experiment. The correction of ${ }^{152 m 1} \mathrm{Eu}$ on the interpretation largely reduces the difference between JEFF-3.1 and PROFIL-2 and shows the agreement between the PROFIL-2 integral experiment and other microscopic measurements. The revaluated neutron-induced total and capture cross sections of ${ }^{151} \mathrm{Eu}$ and ${ }^{153} \mathrm{Eu}$ correspond well with both the microscopic experimental measurements and the PROFIL-2 integral experiment.
\end{abstract}

\section{Introduction}

Neutron cross section is one of the most important quantities in both nuclear physics and nuclear engineering. However, discrepancies can be observed among different evaluated nuclear data libraries. Due to the dispersion among experimental data, it is difficult to determine the "correct" values of cross section. In addition, large experimental uncertainties exist for cross section measurements. A direct consequence is the large uncertainties of the evaluated data.

In comparison with microscopic measurements of cross sections, Post Irradiation Experiments, such as PROFIL, can give lower uncertainties on cross sections. One reason is the accurate measurement of isotopic concentrations using mass spectrometry. Another reason is that the final uncertainty of isotopic concentration is the time accumulated uncertainty of the corresponding cross section. The PROFIL and PROFIL-2 integral experiments $[1,2]$ aim to give feedback to nuclear data evaluations. The interpretation of the PROFIL-2 experiment with JEFF-3.1 library [3] shows that the ratio of calculated to experimental value concerning the neutron capture cross section of ${ }^{151} \mathrm{Eu}$ is 1.980 [2]. This value points out that JEFF-3.1 overestimated about $100 \%$ the neutron capture cross section of ${ }^{151} \mathrm{Eu}$. The present work focuses on the analyses and evaluations of neutron-induced capture cross section of the Europium isotopes ${ }^{151} \mathrm{Eu}$ and ${ }^{153} \mathrm{Eu}$ based on the

*e-mail: shengli.chen@cea.fr
PROFIL-2 experimental data. ${ }^{151} \mathrm{Eu}$ and ${ }^{153} \mathrm{Eu}$ were in the same containers in the PROFIL-2 irradiated experiment.

\section{PROFIL Experiments and Capture Cross Section}

For the neutron-induced capture reaction of isotope ${ }^{A} \mathrm{X}$, the product nucleus is ${ }^{A+1} \mathrm{X}$. The ratios of isotopic concentrations ${ }^{A+1} \mathrm{X} /{ }^{A} \mathrm{X}$ are thus required to show the capture cross sections in the PROFIL experiments. The JEFF-3.1based calculated-to-experimental data $(\mathrm{C} / \mathrm{E})$ and the associated uncertainties of ${ }^{151} \mathrm{Eu}$ and ${ }^{153} \mathrm{Eu}$ are given in Table 1 [2]. The sensitivity of $\mathrm{C} / \mathrm{E}$ to the neutron capture cross section is defined by:

$$
S=\frac{\partial(C / E)}{(C / E)} / \frac{\partial \sigma_{\gamma}}{\sigma_{\gamma}}
$$

where $\sigma_{\gamma}$ refers to the neutron capture cross section. The value of $S$ for each isotope included in the PROFIL experiments has been computed in Ref. [1]. With the sensitivity, the cross section corresponding to the PROFIL experiments can be obtained by:

$$
\sigma_{\gamma, \exp }=\left(1+\frac{1-(C / E)_{J}}{S(C / E)_{J}}\right) \sigma_{\gamma, J}
$$

where the subscript $J$ represents the evaluated data on which the interpretation of the PROFIL experiments are based. Consequently, the energy dependent cross section 
Table 1. C/E values, sensitivities, and uncertainties of the Europium isotopes in the PROFIL-2 experiment

\begin{tabular}{llllll}
\hline & $\mathrm{C} / \mathrm{E}[2]$ & $S[1]$ & $\Delta \phi / \phi$ & $S_{\phi}[4]$ & $\Delta(C / E)_{\phi}$ \\
\hline${ }^{151} \mathrm{Eu}$ & $1.980 \pm 0.032$ & 1.37 & $1.4 \%$ & 1.04 & $1.36 \%$ \\
${ }^{153} \mathrm{Eu}$ & $0.977 \pm 0.002$ & 1.20 & $1.4 \%$ & 0.97 & $1.36 \%$ \\
\hline
\end{tabular}

corresponding the PROFIL experiments should obey:

$\int_{0}^{\infty} \sigma_{\gamma, \exp }(E) \phi(E) d E=\left(1+\frac{1-(C / E)_{J}}{S(C / E)_{J}}\right) \int_{0}^{\infty} \sigma_{\gamma, J}(E) \phi(E) d E$

where $\phi$ stands for the neutron flux. The $\mathrm{C} / \mathrm{E}$ value of a reevaluated neutron capture cross section is thus:

$$
(C / E)_{\text {new }}=\frac{\int_{0}^{\infty} \sigma_{\gamma, \text { new }}(E) \phi(E) d E}{\left(1+\frac{1-(C / E)_{J}}{S(C / E)_{J}}\right) \int_{0}^{\infty} \sigma_{\gamma, J}(E) \phi(E) d E}
$$

Due to the perturbations during the irradiation period, an additional uncertainty of neutron flux $(\Delta \phi / \phi)$ should be taken into account. To study the influence of uncertainties of neutron flux on $\mathrm{C} / \mathrm{E}$ values, the sensitivity to neutron flux is defined as:

$$
S_{\phi}=\frac{\partial(C / E)}{(C / E)} / \frac{\partial \phi}{\phi}
$$

The uncertainty on $\mathrm{C} / \mathrm{E}$ due to the neutron flux is thus:

$$
\Delta(C / E)_{\phi}=S_{\phi}(C / E) /(\Delta \phi / \phi)
$$

The uncertainties of neutron flux, the sensitivities to the neutron flux, and the corresponding uncertainties on $\mathrm{C} / \mathrm{E}$ are given in Table 1.

\section{Methods}

\subsection{Optical model}

In high energy region, the Optical Model (OM) is usually used to calculate total and scattering cross sections. Morillon and Romain have proposed the Dispersive Optical Model Potential (DOMP) based on the dispersive optical model for neutrons with incident energies from $1 \mathrm{keV}$ to $200 \mathrm{MeV}$ [5] as:

$$
\begin{aligned}
U(r, E)= & {\left[V_{v}(E)+i W_{v}(E)\right] f(r, R, a)+\left[V_{s}(E)\right.} \\
& \left.+i W_{s}(E)\right] g(r, R, a)+\left[V_{S O}(E)+i W_{S O}(E)\right] \\
& \times \frac{1}{r}\left(\frac{h}{m_{\pi} c}\right)^{2} g(r, R, a) \mathbf{1} \cdot \boldsymbol{\sigma}
\end{aligned}
$$

where $V$ and $W$ represent the real and imaginary terms of potential and the subscript $V, S, S O$ refer to the Volume, Surface, and Spin-Orbit parts in the potential, respectively. The volume shape function $f$ is a Wood-Saxon form and $g$ is its partial deviation to position $r$ :

$$
\begin{aligned}
& f(r, R, a)=1 /(1+\exp [(r-R) / a]) \\
& g(r, R, a)=-4 a \frac{d}{d r} f(r, R, a)
\end{aligned}
$$

The same radius in Woods-Saxon form is used for different parts in the DOMP. For a deformed nucleus, the radius $R$ in the Woods-Saxon form is given by [6]:

$R=\left(1.295-2.7 \times 10^{-4} A\right) A^{1 / 3}\left[1+\beta_{2} Y_{20}(\Omega)+\beta_{4} Y_{40}(\Omega)\right](f m)$

where $\beta_{2}$ and $\beta_{4}$ are quadrupole and hexadecapole deformation parameters, respectively. $Y_{20}$ and $Y_{40}$ are the corresponding spherical harmonics as a function of the bodyfixed system $\Omega$.

All prior parameters in the optical potential can be found in the Reference Input Parameter Library (RIPL) [7]. The calculations are performed with the nuclear reaction code TALYS [8], in which the optical model code ECIS [9] is included.

\subsection{Statistical model}

The Statistical Model (SM) is used in the present work to calculate the neutron capture cross sections. They are calculated by the Hauser-Feshbach formula [10], in which the transmission coeffcients $T_{n}$ and $T_{\gamma}$ can be obtained with the OM and the Kopecky-Uhl formula [11], respectively. At low incident energy, the gamma-ray transmission coefficient is [12]:

$$
T_{\gamma}=2 \pi \Gamma_{\gamma} / D_{0}
$$

where $\Gamma_{\gamma}$ is the average gamma width, $D_{0}$ refers to the average level spacing. The dependence of $D_{0}$ on neutron energy is determined by the Gilbert-Cameron theory [13].

\subsection{Optimization and uncertainties of parameters}

The CONRAD code [14] is used in the present work for the data assimilation. The Bayes' theorem implicates that the posterior probability density is:

$$
p(\vec{x} \mid \vec{E}, U)=\frac{p(\vec{E} \mid \vec{x}, U) p(\vec{x}, U)}{\int p(\vec{E} \mid \vec{x}, U) p(\vec{x}, U) d \vec{x}}
$$

where vector $\vec{x}$ represents the parameters in the physical model, $\vec{E}$ denotes the experimental data, and $U$ refers as the prior information. Under the hypothesis of Gaussian distribution of the probability density of $\vec{x}$, the maximization of the posterior probability is equivalent to the minimization of the Generalized Least Square (GLS) cost function:

$$
\chi_{G L S}^{2}=\left(\vec{x}-\vec{x}_{0}\right)^{T} M_{x}^{-1}\left(\vec{x}-\vec{x}_{0}\right)+(\vec{C}-\vec{E})^{T} M_{E}^{-1}(\vec{C}-\vec{E})
$$

where $\vec{x}_{0}$ represents the vector containing prior values, $\vec{C}$ and $\vec{E}$ denote the calculated and experimental data, respectively. $M_{x}\left(M_{E}\right.$ resp.) stands for the covariance matrix of $\vec{x}$ ( $\vec{E}$ resp.) [15]. The fitted parameters in the present work are nuclear deformation parameters $\beta_{2}$ and $\beta_{4}$, and average gamma width $\Gamma_{\gamma}$. The experimental data are the $s$-wave neutron strength function $S_{0}$ [16], total cross sections collected in EXFOR [17], and the integral data of the PROFIL-2 experiment (with $2 \sigma$ uncertainty for the conservative consideration). 
The parameters contained in the vector $\vec{x}$ are observable parameters. On the other hand, the nuisance parameters have also contributions to the posterior covariance matrix of observable parameters $\vec{x}$. The posterior covariance matrix among observable parameters can be finally expressed by [18]:

$$
\Sigma_{11}=M_{x}+\left(G_{x}^{T} G_{x}\right)^{-1} G_{x}^{T} G_{\theta} M_{\theta} G_{\theta}^{T} G_{x}\left(G_{x}^{T} G_{x}\right)^{-1}
$$

where the matrices $G$ contain partial derivation of calculated values. For $k=x, \theta$,

$$
G_{k,(i, j)}=\partial C_{i} / \partial k_{j}
$$

\section{Results and discussion}

The fitted model parameters $\left(\beta_{2}, \beta_{4}\right.$, and $\left.\Gamma_{\gamma}\right)$ and the model based calculated data $\left(S_{0}\right.$ and $\left.\mathrm{C} / \mathrm{E}\right)$ are given in Table 2.

\subsection{Total and capture cross sections of ${ }^{153} \mathrm{Eu}$}

The results in Table 2 show that the model parameters determined for ${ }^{153} \mathrm{Eu}$ can well reproduce the $S_{0}$ and the capture cross section is in good agreement with the PROFIL2 experiment. The total and capture cross sections are shown in Figure 1. Prior and posterior refer to the cross section calculated by the prior and posterior parameters, respectively. The reevaluated total cross sections correspond better to the experimental data than JEFF-3.1. The capture cross sections have little difference to JEFF-3.1. Both JEFF-3.1 and the reevaluated cross sections are in good agreement with experimental data.

\subsection{Capture cross sections of ${ }^{151} \mathrm{Eu}$}

Table 2 shows that the fitted $\Gamma_{\gamma}$ is much smaller than the experimental one. As indicated previously, the $1.980 \mathrm{C} / \mathrm{E}$ value ${ }^{152} \mathrm{Eu} /{ }^{151} \mathrm{Eu}$ reveals that the capture cross section corresponding to the PROFIL-2 experiment is about half of the data in JEFF-3.1. In addition, the posterior capture cross section is largely lower than experimental microscopic cross sections (Figure 2). In this context, further analysis is necessary for the capture cross section of the ${ }^{151} \mathrm{Eu}$.

In fact, there are two metastable isotopes of ${ }^{152} \mathrm{Eu}$, which have 9 hours $\left({ }^{152 m 1} \mathrm{Eu}\right)$ and 96 minutes $\left({ }^{152 m 2} \mathrm{Eu}\right)$ half-lives, respectively (shown in Figure 3). EAF-2010 [20] points out $34.4 \%$ reaction yield of ${ }^{152 \mathrm{ml}} \mathrm{Eu}$. The $\beta$ decay or the electron capture of ${ }^{152 m 1} \mathrm{Eu}$ produces ${ }^{152} \mathrm{Gd}$ or ${ }^{152} \mathrm{Sm}$. However, only the ground state ${ }^{152} \mathrm{Eu}$ is included in ERANOS code [21], which was used to interpret the PROFIL experiments. The $\mathrm{C} / \mathrm{E}$ value for the capture cross section is lower if ${ }^{152 m 1} \mathrm{Eu}$ is taken into account.

Using the branching ratio of ${ }^{152} \mathrm{Eu}$, noted as $R_{0}$, the corrected $\mathrm{C} / \mathrm{E}$ is:

$$
(C / E)_{c}=R_{0}(C / E)_{\text {ERANOS }}=1.299
$$

Accordingly, the prior value should be $(C / E)_{\text {prior }}=0.941$. $1.62 \%$ experimental uncertainty and $2.72 \%(2 \sigma)$ complementary fluence uncertainty lead to $3.2 \%$ uncertainty on
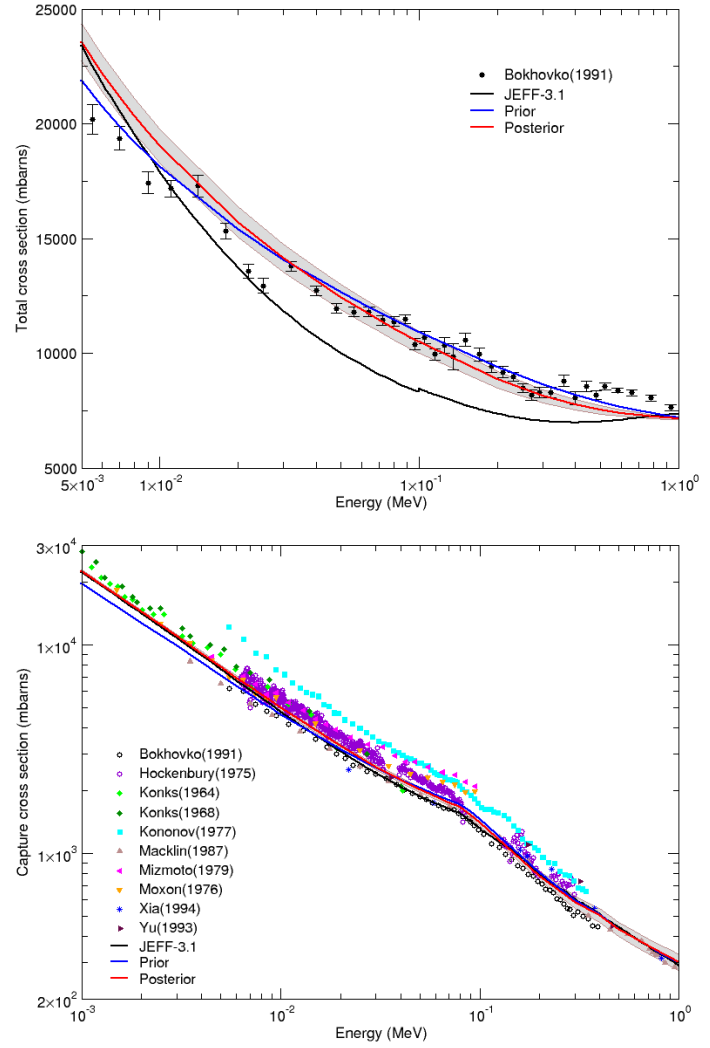

Figure 1. Total cross section and capture cross section of ${ }^{153} \mathrm{Eu}$ with $1 \sigma$ uncertainty of the posterior cross sections (in grey)

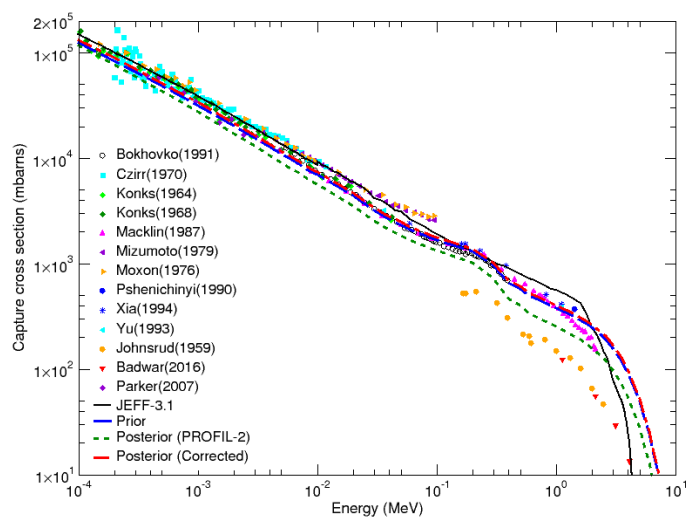

Figure 2. Neutron capture cross section of ${ }^{151} \mathrm{Eu}$

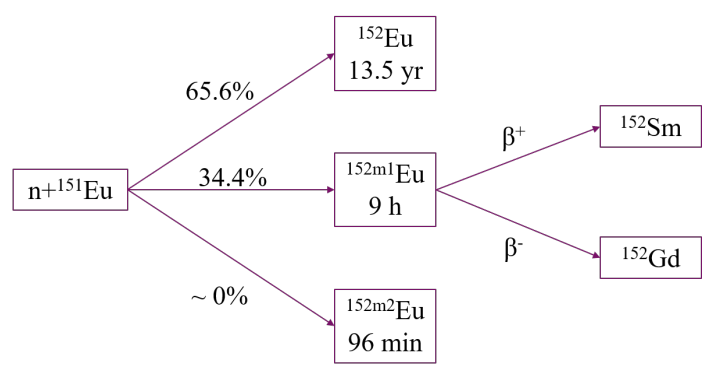

Figure 3. Scheme of $n+{ }^{151}$ Eu capture reactions 
Table 2. Experimental (first 2 lines) and posterior (last 2 lines) model parameters and evaluated data

\begin{tabular}{llllll}
\hline Isotope & $\beta_{2}$ & $-\beta_{4}$ & $\Gamma_{\gamma}[19]$ & $10^{-4} S_{0}[19]$ & $C / E[2]$ \\
\hline${ }^{151} \mathrm{Eu}$ & 0.2520 & 0.1500 & $0.091 \pm 0.009$ & $3.4 \pm 0.3$ & $1.980 \pm 0.032$ \\
${ }^{153} \mathrm{Eu}$ & 0.1727 & 0.0076 & $0.093 \pm 0.003$ & $2.5 \pm 0.2$ & $0.977 \pm 0.002$ \\
\hline${ }^{151} \mathrm{Eu}$ & $0.210 \pm 0.009$ & $0.080 \pm 0.008$ & $0.057 \pm 0.016$ & $3.41 \pm 0.30$ & $1.037 \pm 0.063$ \\
${ }^{153} \mathrm{Eu}$ & $0.231 \pm 0.001$ & $0.098 \pm 0.034$ & $0.093 \pm 0.011$ & $2.50 \pm 0.03$ & $1.013 \pm 0.038$ \\
\hline
\end{tabular}

the C/E. The prior result (JEFF-3.1 resp.) is thus in good agreement with the PROFIL-2 experiment within $2 \sigma$ ( $1 \sigma$ resp.) uncertainty.

By adjusting the nuclear deformation parameter $\beta_{2}$ in optical calculation according to the compiled value of $S_{0}$ [19], the value 3.41 for $10^{-4} S_{0}$ is obtained with the fitted $\beta_{2}$ value 0.210 . With the adjustment of $\Gamma_{\gamma}$ to achieve $(C / E)_{\text {posterior }}=1$, the posterior neutron capture cross section at $30 \mathrm{keV}$ is $3.368 \mathrm{barn}$, which corresponds better with the compiled value in Ref. [19] (3.458 \pm 0.102 barn) than prior cross section (3.169 barn) and JEFF-3.1 (4.200 barn).

\section{Conclusions}

The total and capture cross sections of ${ }^{153} \mathrm{Eu}$ are reevaluated according to the PROFIL-2 experiment and compiled value of $s$-wave neutron strength function and experimental total cross section from EXFOR. The reevaluated cross sections are in good agreement with the above reference data. The large overestimation of the capture cross section of ${ }^{151} \mathrm{Eu}$ according to the previous interpretation of the PROFIL-2 experiment is due to the lack of ${ }^{152 \mathrm{ml}} \mathrm{Eu}$ in the ERANOS code. Further analysis in the present work shows that the prior result and JEFF-3.1 are in good agreement with the PROFIL-2 experiment within respectively $2 \sigma$ and $1 \sigma$ uncertainty. The reevaluated capture cross section of ${ }^{151} \mathrm{Eu}$ corresponds well with other reference data.

\section{References}

[1] J. Tommasi, E. Dupont, and P. Marimbeau, Nucl. Sci. Eng., 154(2), 119-133 (2006)

[2] J. Tommasi and G. Noguere, Nucl. Sci. Eng., 160(2), 232-241 (2008)

[3] A. Koning et al., The JEFF-3.1 nuclear data library. OECD (2006)
[4] E. Privas, P. Archier, C. D. S. Jean, G. Noguere, and J. Tommasi, Nucl. Sci. Eng., 182(3), 377-393 (2016)

[5] B. Morillon and P. Romain, Phys. Rev. C, 70(1), 014601 (2004)

[6] P. Romain and J. P. Delaroche, Specialists' Meeting on the Nucleon Nucleus Optical Model up to $200 \mathrm{MeV}$, Bruyeres-le-Chatel, France (1996)

[7] R. Capote et al., Nucl. Data Sheets, 110(12), 31073214 (2009)

[8] A. J. Koning, S. Hilaire, and M. C. Duijvestijn, ND2017, 211-214 (2007)

[9] J. Raynal, NEA/Paris, France (2003)

[10] W. Hauser and H. Feshbach, Phys. Rev., 87(2), 366 (1952)

[11] J. Kopecky and M. Uhl, Phys. Rev. C, 41(5), 19411955 (1990)

[12] B. J. Allen et al., Neutron Radiative Capture, 3 Neutron Physics and Nuclear Data in Science and Technology (1984)

[13] A. Gilbert and A. Cameron, Can. J. Phys., 43(8), 1446-1496 (1965)

[14] P. Archier et al., Nucl. Data Sheets, 118, 488-490 (2014)

[15] S. Chen et al., EPJ Web Conf., 211, 07002 (2019)

[16] K. K. Seth, Nucl. Data Sheets Sect. A, 2(3), 299-332 (1966)

[17] N. Otuka et al., Nucl. Data Sheets, 120, 272-276 (2014)

[18] G. Noguere, P. Archier, C. D. S. Jean, and B. Habert, Nucl. Sci. Eng., 172(2), 164-179 (2012)

[19] S. F. Mughabghab, Atlas of Neutron Resonances: Resonance Parameters and Thermal Cross Sections. $Z=1-100$ (Elsevier, 2006)

[20] J.-C. Sublet et al., EASY Doc. Ser. CCFE (2010)

[21] J. M. Ruggieri et al., Proceedings of ICAPP 06, Reno, NV USA, 2432-2439 (2006) 